\title{
La Formación de Docentes para la Educación Inclusiva. Un Reto desde la Universidad Nacional de Educación en Ecuador
}

\section{Teacher Training for Inclusive Education. A Challenge from the National University of Education in Ecuador}

\author{
José Ignacio Herrera ${ }^{1 *}$ \\ Ángeles Parrilla ${ }^{2}$ \\ Antonia Blanco ${ }^{2}$ \\ Geycell Guevara ${ }^{1}$ \\ ${ }^{1}$ Universidad Nacional de Educación, Ecuador \\ ${ }^{2}$ Universidad de Vigo, España
}

\begin{abstract}
Una demanda del sistema educativo ecuatoriano es la formación de docentes, comprometidos con las transformaciones de la sociedad; con altos valores humanistas, éticos, innovadores e investigativos y generadores de ambientes para atender la diversidad. Por lo que en el presente artículo se propone reflexionar acerca del proceso de formación de los profesionales de la educación para conducir prácticas inclusivas desde el modelo de la Universidad Nacional de Educación (UNAE). Este trabajo parte de estudios realizados acerca de la formación del profesional de la educación con énfasis en el profesorado de la educación especial, así como de las demandas que la sociedad ecuatoriana plantea a sus instituciones educativas para que sean más inclusivas y abiertas a la diversidad. Estas premisas permitieron diseñar e implementar la carrera de Licenciatura en Educación Especial con enfoque inclusivo desde la Universidad Nacional de Educación (UNAE), donde se acumulan interesantes experiencias.
\end{abstract}

Descriptores: Formación del personal docente; Formación de docentes de educación especial; Escuela especial; Profesor especializado; Ayuda educativa.

\begin{abstract}
The training of teachers committed to their times, with high humanistic, ethical, innovative and investigative values, generators of environments for attention to diversity is a demand of the Ecuadorian educational system, the objective of the article is to propose a reconsideration of the process of formation of professionals for attention to diversity, capable of conducting inclusive educational practices from the pedagogical model of UNAE. The work makes sense from the trend studies on teacher training, with emphasis on teachers for special education and support schools, as well as the demands that Ecuadorian society poses to their educational institutions to make it every day more inclusive and open to diversity. Based on these premises, a Degree in Inclusive Education was designed and implemented by the National University of Education (UNAE), where interesting experience is accumulated in the training process of professionals.
\end{abstract}

Keywords: Training of teaching staff; Special teacher education; Special school; Special education teacher; Educational assistance. 


\section{Introducción}

Un anhelo de los sistemas educativos a nivel internacional es la formación de docentes para trabajar con la diversidad de estudiantes en el marco de un aula inclusiva, donde se promueva la igualdad y la justicia social. Al respecto, la Agenda 2030 expresa que se debe: garantizar una educación inclusiva y equitativa de calidad y promover oportunidades de aprendizaje permanente para todos.

La educación inclusiva implica un replanteo de los procesos de formación del profesorado. Los debates, sobre el tema, se encuentran en informes y documentos internacionales que recogen estas demandas, entre las que se encuentran: "Guías para las políticas sobre la inclusión en educación” (UNESCO, 2009); el World Report on Disability (WHO, 2011), el informe de la OCDE (2010) sobre Educación de profesores para la diversidad y el trabajo de la Agencia Europea para el Desarrollo de las Necesidades Educativas Especiales (NEE) Formación del profesorado para la educación inclusiva en Europa (EADSNE, 2012).

Si bien, América Latina se incorporó con cierto desfase al movimiento inclusivo, este tema ha sido tratado de forma prioritaria en los últimos años por los países que lo componen. Informes como la Xx Cumbre Iberoamericana de Jefes de Estado y de Gobierno (OEI, 2010); el documento de la UNESCO (2012) para la elaboración de políticas de inclusión en América Latina y el Caribe y los trabajos de Payá (2010), Blanco y Duk (2011), Vaillant (2011, 2013) así lo confirman. En ellos se analizan las políticas y prácticas inclusivas en la región y la formación de profesores para la educación básica y la inclusión.

El informe de la UNESCO (2015a) referido a los progresos que desde el año 2000 al 2015 se logró en la Educación para Todos en Ecuador (Revisión Nacional 2015 de la Educación para Todos), insiste en que aún no son suficientes los avances hacia una educación inclusiva. La identificación de barreras y la búsqueda de procesos educativos, formativos, políticos para el desarrollo de una educación y sociedad más inclusiva, innovadora y reflexiva sigue siendo una cuestión pendiente. Estos aspectos han sido refrendados en el Foro Mundial por la Educación de 2015 (UNESCO, 2015b).

En el análisis acerca de la situación del profesorado para enfrentar la educación inclusiva, se reconoce la necesidad de fortalecer su formación para esta tarea. Durante su implementación en el Ecuador, con el Plan Decenal de Educación (2006-2015), estos nudos críticos se han superado. La política pública educativa incrementó las coberturas en la Educación Inicial y Básica. Además, se ha elevado la calidad del sistema educativo, los niveles de gobernanza y la eficacia institucional.

Se concibe el Plan Nacional de Desarrollo 2017-2021 y la Estrategia de desarrollo del Plan Toda una Vida, Ecuador 2030 y se declara que: "Ecuador promoverá la solidaridad y el respeto a la diversidad" (p. 32). En este documento, "se reconoce y celebra la igualdad en la diversidad que poseen los diferentes sujetos de derechos" (p. 36). Para su cumplimiento, se desplegaron acciones, por lo que la inclusión educativa llegó al 87\% de la población en edad escolar con Necesidades Educativas Especiales (NEE), asociadas o no a una discapacidad. Además, se crearon a nivel nacional 140 Unidades de Apoyo a la Inclusión (UDAI) para fortalecer la educación especializada e inclusiva y garantizar el acceso, la permanencia, la participación y el aprendizaje de los niños y jóvenes con NEE.

Se puede decir que Ecuador cuenta con una política pública que define y orienta la educación inclusiva en las instituciones educativas. Para dar cumplimiento a estas 
políticas, se emiten orientaciones para los currículos y adaptaciones curriculares que atienden a los niños con NEE. Aunque se habla de flexibilidad curricular para atender la diversidad, aún se sigue orientando desde la discapacidad.

Este discurso inclusivo, todavía está permeado por ideas asistencialistas e integradoras. Se piensa que la solución se encuentra en etiquetar y clasificar a los niños para enviarlos a escuelas especiales o consultas de psicólogos o terapeutas. Por otro lado, se cuenta con docentes interesados en mejorar sus prácticas inclusivas, pero que no poseen las herramientas pedagógicas para hacerlo.

El Sistema Nacional de Educación tiene un déficit aproximado de 160.000 vacantes, proyectadas hasta el 2030, según el diagnóstico del expediente de creación de la UNAE (2013). Se pretende su inserción cualificada en el ámbito del Sistema Nacional de Educación para brindar educación de calidad, con enfoque inclusivo, de equidad y respetando las características de pluriculturalidad y multilingüismo de los individuos.

En el informe Ser Maestro, 2014, estudio realizado sobre el desempeño docente del magisterio ecuatoriano, se refleja la necesidad de desarrollar las competencias, habilidades y saberes de los procesos de enseñanza y aprendizaje inclusivos. Un $45 \%$ de los docentes manifiestan que tienen una insuficiente formación en educación especial y en educación para la ciudadanía, cuestiones directamente vinculadas a la educación desde el enfoque inclusivo.

Los estudios exploratorios sobre pertinencia y perfil de egreso para la carrera de Educación Especial con enfoque inclusivo (Parrilla, Blanco y Herrera, 2015), realizados por la Universidad Nacional de Educación (UNAE), constatan que existen carencias relacionadas con el trabajo en equipos y redes, con los procesos de identificación de barreras para el aprendizaje y la participación, con el diseño de currículos diversificados y universales para el aprendizaje y con el manejo didáctico en un aula inclusiva.

La UNAE es una institución pública, que tiene la misión de formar los nuevos docentes que necesita el país para desarrollar y transformar el sistema educativo de Ecuador. Posee un modelo pedagógico que se caracteriza por trasladar la docencia del campo de la explicación al de la tutorización, para que cada estudiante se entienda a sí mismo y desarrolle sus proyectos vital y profesional, así como las competencias para ser un docente del siglo XXI y de la era digital (Pérez Gómez, 2013).

Estos argumentos conllevaron a la reflexión del proceso de formación de los profesionales de la educación con el fin de prepararlos para la atención a la diversidad en el sistema educativo ecuatoriano y para conducir prácticas inclusivas, sustentadas en el modelo pedagógico de la UNAE.

\section{Tendencias en la formación del profesional docente. Un acercamiento al docente de la educación especial}

La formación del profesorado históricamente ha contado con disímiles modelos, tradiciones o perspectivas de formación. A su vez, estos presentan en su interior, variadas manifestaciones o diversificaciones.

Según Feiman-Nemser (1990) la tradición académica se centra en la figura del profesor y en su actividad docente, considerándolo como un experto en la materia que enseña y ofrece 
un conjunto de normas de comportamientos profesionales para su aplicación práctica. Por su parte, la tradición tecnológica concibe a los profesores como técnicos y la finalidad es capacitarlos en habilidades que han demostrado ser eficaces para el desarrollo profesional.

Shön (1992) describe la perspectiva práctico-crítica como aquella que entiende al profesor como el profesional que debe aprender a resolver problemas en situaciones de incertidumbre, singularidad y conflictos. La finalidad es promover la formación y el desarrollo profesional a través de la práctica.

Pese a que no todas las clasificaciones son iguales (Marcelo, 1995; Pérez Gómez, 2010), en el modelo pedagógico de la UNAE se plantea la formación desde la concepción de los profesionales de la educación como prácticos-reflexivos y críticos.

Desde la perspectiva práctica de formación del profesorado (Pérez Gómez, 1992) se asume que los procesos educativos son multidimensionales, complejos, imprecisos e inciertos, como cualquier interacción humana. Por tanto, los profesores deben adquirir saberes para trabajar con la complejidad y el cambio; les brinde la posibilidad de flexibilizar sus herramientas de trabajo y adaptarse a situaciones cambiantes. Shön (1987) también señala que el pensamiento práctico está compuesto por tres conceptos o fases: el conocimiento en la acción, la reflexión en y durante la acción y la reflexión sobre la acción. Desde este enfoque se propone una relación no jerárquica entre la teoría y la práctica, siendo el saber práctico quien debe guiar al saber teórico.

La tradición reconstruccionista social de formación del profesorado (Liston y Zeichner, 1993) o la perspectiva de reflexión en la práctica para la reconstrucción social (Pérez Gómez, 1992) asumen la enseñanza como una actividad creativa que demanda al profesorado estar capacitado para la improvisación, la reflexión en la acción y la investigación de su práctica diaria. Además, subraya el carácter político de la educación; pues en su seno tienen lugar dinámicas de poder.

Bajo la idea de preparar a los futuros docentes como intelectuales y trabajadores de y con la cultura (Giroux, 2001), es que Pérez Gómez (1992, p. 423) declara las características que debe tener un programa de formación del profesorado. Plantea que debe proporcionar un bagaje cultural de orientación política y desarrollar en el alumnado la capacidad de reflexión crítica respecto a la sociedad, al aula-centro y a sí mismo. También debe favorecer el desarrollo de actitudes que requieran un compromiso político. Estos referentes priorizan la práctica pre-profesional y los métodos inductivos-deductivos en el proceso de formación del profesorado.

Las propuestas formativas se orientan según determinados valores, conceptos y culturas sobre la escuela, los profesores y el cambio (Marcelo, 1995). No es lo mismo formar a un profesional desde un enfoque personalista que hacerlo desde un enfoque tecnológico o desde uno artesanal o crítico. Por lo que, a continuación, se mencionan diferentes tipos de orientaciones (Parrilla, 1997, 2015) para la formación de profesionales de la educación especial

\section{El profesor como práctico}

Enfatiza en las prácticas tradicionales y considera la educación especial como un trabajo artesanal en el que la experiencia se identifica como fuente del aprendizaje del profesor. Se destaca la práctica como principal estrategia formativa del profesional. Esta postura ha recibido fuertes críticas por reducir la formación a experiencias prácticas. Florian (2008), 
opina que en la formación inicial se han creado largos períodos prácticos, lo representa reducir la formación inicial a un aprendizaje práctico, sin referentes teóricos que permitan reconstruirla críticamente.

\section{El profesor como especialista}

Concibe la formación de profesionales como un proceso de transmisión de conocimientos científicos y culturales cuya meta es dotarlos de una formación orientada al dominio de los conceptos y la estructura disciplinar de la materia que enseñan. Las críticas se centran en que la especialización puede tener a una visión mutilada y sectorial, que se contrapone con una visión general de los problemas.

\section{El profesor como técnico: un sujeto con destrezas}

Se entiende al profesor como un técnico que domina el conocimiento científico producido por otros y que lo convierte en reglas de actuación, es decir, un especialista que emite «recetas» acerca de cómo actuar, tal y como sucede en la formación de la educación especial tradicional. Este modelo ha sido criticado por su incapacidad para dirigir la práctica y ayudar a su reconstrucción (Martínez Bonafé, 1995).

\section{El profesor como persona}

Aquí se opta por una formación más atenta a las características individuales y personales. Se concibe la educación como dependiente a cada caso, de biografías particulares, de personalidades concretas. Los programas formativos desarrollados bajo esta opción hacen hincapié en el compromiso personal de los profesores con la sociedad.

\section{El profesor como práctico-reflexivo}

Plantea la necesidad de superar la racionalidad técnica y propone una orientación crítica en la formación. Se concibe la reflexión como instrumento básico de aprendizaje e incorpora un compromiso ético y social de búsqueda de prácticas educativas más justas y democráticas. Se diseñan programas formativos basados en currículos abiertos, orientados a la indagación, que enfatizan los elementos cognitivos de la actividad del profesional de apoyo, así como la construcción del conocimiento que supone dicha actividad.

Cada una de estas concepciones va a influir de forma determinante en los contenidos, métodos y estrategias para formar a los profesores de educación especial. Aunque ninguna de las orientaciones analizadas explica y comprende, en su totalidad, la complejidad de la formación del profesorado de apoyo como "aquel profesional ocupado del refuerzo en los centros ordinarios a tiempo parcial, sin grupo propio y atendiendo a alumnos que continúan de forma ordinaria en el grupo y aula a los que pertenece" (Moya, 2012, p. 72). Si bien, algunas muestran una mayor sensibilidad a las cuestiones de la diversidad, su bondad no es independiente del proyecto formativo que pretenden desarrollar. El ideal estaría en la complementariedad que se logra desde todas ellas.

\section{La formación de docentes para la educación inclusiva. Un proceso en construcción}

En la historia de la formación de los docentes para la educación especial se cuestiona si lo ideal es tener una doble titulación (primero general y luego especial) o directamente estudiar la carrera de educación especial (una única titulación). 
¿Deben estos profesores obtener una doble titulación, primero general y luego especial? o ¿es posible que su formación se conciba directamente como especial, es decir, en una única titulación? (Parrilla, 2015). Las consecuencias de guiarse por una u otra opción son diferentes en el ejercicio de los profesores.

Existen diferentes modelos de formación para los docentes de educación especial. Un primer modelo recibe una formación general y posteriormente una especializada. Un segundo que se desdobla en una formación generalista y otra para especialistas. Por último, el que ofrece una enseñanza especializada como formación única durante su carrera a los profesores de educación especial.

Los riesgos de los modelos que separan a los profesionales en su formación inicial han sido planteados por numerosos autores (Forlin, 2010, 2012; UNESCO, 2012) y señalan que: se perpetúa para el profesor de enseñanza general la idea de que la inclusión es cosa de otros (por eso se separan); se abona el terreno para unas difíciles relaciones interprofesionales (a los profesores tutores/generalistas les queda claro que los profesores de educación especial o de apoyo, que son los que les orientan y guían en su tarea, no conocen el mundo de la enseñanza general) y se perpetúa el universo referencial segregado -aunque solo sea en términos formativos- de los distintos tipos de profesionales.

Según Abendaño Briceño (2004) en Ecuador la formación de docentes con nivel de licenciatura se desarrolla en las universidades de cada provincia de Ecuador. El 90\% de las Facultades tienen carreras universitarias que confieren el título de Licenciado en Ciencias de la Educación, en la especialización de Educación Parvularia o Primaria, aunque algunas conceden el título de Profesor Primario o Parvulario. Estas tienen una duración de cuatro años u ocho semestres de estudios, pero la formación que se ofrece para la atención a la diversidad se limita al trabajo con las NEE.

En ninguna se ofertaba la Licenciatura en Educación Especial, hasta que en el 2015 se abre esta carrera en la UNAE y tiene la misión de mejorar la formación docente en todas las áreas del conocimiento y, especialmente, en la educación inclusiva como requisito fundamental para la mejora de la calidad y equidad en todas las áreas de la sociedad.

\section{La formación categórica, la formación no categórica y la formación inclusiva}

La formación categórica (Mitler, 1993; Wang, Reynolds y Walberg, 1987) supone la formación de los profesionales basada en categorías de déficits (tanto en torno a la organización del currículo, como a las especializaciones). Esta formación enfatiza en la tecnificación del profesor de acuerdo a cada una de estas categorías.

Desde este planteamiento, un currículo formativo da lugar a la obtención de titulaciones o especializaciones como profesor de educación especial para niños ciegos, sordos o retrasados mentales, etc. Es una formación acorde con el modelo educativo especial basado en los déficits, que va quedando atrás desde las prácticas inclusivas donde se censura la disonancia del modelo formativo categórico. Pullin (2008) y Riddell (2014) son autores que señalan la disfuncionalidad de este modelo formativo en la formación del profesorado de la inclusión.

Hardman y McDonnel (2008) señalan que los modelos de formación categóricos no llegan a cubrir todas las necesidades de los alumnos con discapacidades (profesionales 
especializados en categorías deficitarias: psicomotricidad, sensoriales, emocionales y de la conducta o del lenguaje), refuerzan la tendencia al etiquetaje y enfatizan en el diagnóstico psicológico más que en aspectos educativos de la programación.

Frente al enfoque anterior, se desarrollan propuestas como la no categórica (también llamada cross-categórica, multicategórica o polivalente). Su objetivo es formar al profesorado en habilidades, destrezas y competencias específicas e indispensables para la consecución de programas de integración escolar. Las competencias propuestas hacen referencia a contenidos, habilidades o estrategias didácticas para el desarrollo del currículo, la gestión de la clase y la detección de NEE (Parrilla, 1997).

Blackhurst, Bott y Cross (1987) y Cuomo (1994) señalan como ventajas de la formación polivalente o no categórica que potencia un desarrollo de la atención educativa al alumno y de los servicios educativos centrados en las necesidades individuales y no en su condición deficitaria. Además ofrece una formación que prepara al profesor para atender a niños con una gran variedad de características.

La tercera de las orientaciones es la formación inclusiva, formación en diversidad o pedagogía inclusiva (Florian, 2008, 2010, 2013, 2014a Spratt y Florian, 2013). Esta insiste en desviar el foco desde los alumnos hasta la enseñanza. Además proporciona apoyo para todos, se reconocen las diferencias individuales, pero la idea es la propuesta única y diversa. Por tanto supone un cambio en el pensamiento pedagógico, pues se crean oportunidades de aprendizajes ricos y accesibles para todos los alumnos.

Según Florian (2014b) se necesitan nuevas formas de trabajo en la educación especial basadas en el apoyo y promoción del aprendizaje para todos los alumnos. Para ello, son fundamentales la participación de todos los estudiantes y el trabajo colaborativo.

Parrilla (1997) realiza un análisis sobre la formación de docentes y la posibilidad de articularla en torno a áreas de intervención, según las NEE de los educandos o de formar un especialista capaz de trabajar desde la diversidad.

La formación en torno a áreas de intervención en una determinada etapa o nivel de enseñanza, capacita al docente para trabajar con problemas relativos a las NEE. Como se aprecia, es una propuesta muy asistencialista, pues concibe a los sujetos con NEE como personas que padecen una enfermedad y se crean instituciones para cada tipo de necesidad. Aquí la diversidad se entiende desde las NEE y no responde a las expectativas de la escuela del siglo XXI.

El énfasis en el alumno como sujeto de enseñanza o en el currículo es, sin dudas, otro de los debates acerca del diseño de propuestas formativas. Deja traslucir propuestas referidas a una formación orientada a la intervención individual del alumno o a una intervención más amplia dirigida a la escuela y desarrollada a través del currículo. (Marcelo y LópezYáñez, 1997; Parrilla, 1996).

Otra posición es la formación que apuesta por una intervención educativa dirigida a la escuela y realizada a través del currículo. Se asume que las dificultades de los alumnos surgen a partir de la interacción de factores que, en su conjunto, demuestran un problema institucional, concretado en términos curriculares.

Por tanto, se aprecian dos posiciones en la formación de docentes para la Educación Especial: el enfoque formativo tradicional, centrado en el diagnóstico de dificultades y la intervención en los contextos de una escuela especial y el enfoque inclusivo que forma 
docentes para responder a la diversidad de estudiantes en el contexto de la educación regular (Ainscow, 2007, 2015; Ainscow y Sandil, 2010). Esta última posición se asume en la UNAE para diseñar y aplicar la carrera en Educación Especial con enfoque inclusivo.

A pesar de que ya existen múltiples e interesantes experiencias en la formación de docentes para el trabajo con la diversidad, estas propuestas no han perdido novedad, aun cuando se aprecia cierta resistencia al cambio y la inclusión sigue asociándose a los sujetos con NEE. Estas concepciones continúan enfatizando en las carencias, sin tomar en cuenta las barreras que imponen los contextos para el aprendizaje y la participación. Por lo que se sigue dando prioridad a las adaptaciones curriculares, en vez de pensar en un currículo para la diversidad.

\section{La formación de docentes para la educación inclusiva desde la Universidad Nacional de Educación}

Para transformar el Sistema Educativo Nacional de Ecuador se creó la UNAE, el 19 de diciembre de 2013. Esta es una institución pública considerada "emblemática" y tiene la misión de formar los nuevos docentes que necesita el país.

La carrera de Educación Especial con enfoque inclusivo tiene como objetivo la formación de docentes de excelencia para la dirección del proceso de enseñanza-aprendizaje con orientación inclusiva, según el acuerdo del Consejo de Educación Superior CES ACU-SQ017-No 112-2016 (Malla de la Carrera de Educación Especial con enfoque inclusivo, 2016).

En la modelación de la carrera se tomaron en cuenta los horizontes epistemológicos que sustenta el modelo pedagógico de la UNAE y que rompe con viejos esquemas de enseñanzaaprendizaje. Este proceso se trasforma en un inter-aprendizaje, en el que aprender a aprender se convierte en el camino que guía la comunidad. Desde esta perspectiva, todos son aprendices que crean, re-crean, de-construyen, construyen el mundo, rompen esquemas disciplinarios para vivir y poder ser.

Los núcleos básicos de las disciplinas que sustentan la carrera de Educación Especial con enfoque inclusivo se encuentran en: los sujetos, contextos y sistemas socio educativos; el aprendizaje humano; el currículo y su gestión; los ambientes, procesos y resultados de aprendizaje; las comunidades de aprendizaje e intervención educativa.

Basado en el modelo institucional de la UNAE, se pretende que la carrera de Educación Especial con enfoque inclusivo transforme las prácticas pedagógicas y garantice la equidad e inclusión, la calidad e innovación, la formación holística, la generación de conocimiento, la ciudadanía democrática para el Buen Vivir, la interculturalidad y diálogo de saberes y el enfoque de género e integralidad.

\section{Los problemas de la profesión en la carrera de Educación Especial con enfoque inclusivo. Orientaciones metodológicas para su transformación}

Para transformar los problemas de la profesión, la carrera identifica un grupo de orientaciones metodológicas: 


\section{Eje investigación y praxis}

La investigación y la práctica como ejes integradores. La práctica como investigación en la acción, integrada a la vinculación para actuar profesionalmente con un enfoque de resolución de problemas, investigación sobre la práctica, permanente reflexión individual y con otros, sólida formación disciplinar y compromiso ético con la educación como derecho de todos.

La carrera se sustenta en la investigación gestionada por todo el equipo de docentesinvestigadores, donde el contacto con la realidad educativa ocurre desde el mismo inicio de los estudios, garantizando un proceso de aprendizaje experiencial y activo.

\section{Aprender a aprender}

Constituye el referente pedagógico de las carreras de la UNAE. Se estructura en una organización concebida desde la complejidad sistémica, el diálogo de saberes (interactividad, interculturalidad, interdisciplinariedad), el aprendizaje en contextos de aplicación para transformar la realidad. Se integran las redes sociales virtuales, las plataformas digitales y promueve la didáctica invertida (flipped classroom). Se reserva el espacio del aula y el tiempo presencial para investigar, profundizar, practicar, proyectar, resolver dudas, orientar de manera personalizada y fomentar la cooperación y el debate.

\section{Investigación formativa}

Se asume la investigación formativa y generativa. La primera, como parte de los procesos formativos, entendida como construcción de conocimientos en contextos profesionales y/o educativos que se orienta hacia la apropiación de saberes disciplinares. Contribuye a la búsqueda de soluciones creativas y formas de intervención.

La investigación-reflexión-acción es parte fundamental del proceso educativo para que los estudiantes asuman la responsabilidad sobre su aprendizaje, construyan un conocimiento significativo, desarrollen la metacognición y puedan continuar aprendiendo a lo largo de la vida.

Los ciclos de formación culminan con la presentación de proyectos de investigación y ejercicios integradores de saberes. Se valora la formación de competencias para la dirección de los procesos educativos inclusivos, donde los aprendizajes adquiridos en el aula de clases se corroboran en la práctica aplicando metodologías y técnicas de investigación científica. Estas experiencias se consolidan en cada ciclo de formación en función de los proyectos de grado para culminar sus estudios universitarios.

\section{La integración curricular entre asignaturas para la implementación de redes de aprendizajes, proyectos de integración de saberes y prácticas pre-profesionales}

Las asignaturas se integran por ciclos mediante los campos de formación. Estos se desarrollan a través de la teoría, las prácticas pre-profesionales y la investigación a lo largo del currículo y por los núcleos problémicos que hacen énfasis en:

- La Unidad Básica: se observa y reflexiona sobre políticas educativas, con énfasis en las políticas de inclusión educativa; acerca del aprendizaje de sí mismo y de 
los otros; de acuerdo al sujeto, a los contextos y a los sistemas socio-educativos; a través de los proyectos de integración de saberes.

- La Unidad Profesional: se centra en el análisis y sistematización de experiencias de enseñanza-aprendizaje, mediante el núcleo problémico: qué y cómo enseñar; el currículo y su gestión; los ambientes, procesos y resultados de aprendizaje.

- La Unidad de Titulación: hace énfasis en la concreción y evaluación de los procesos de enseñanza-aprendizaje desde el currículo y el núcleo problémico: comunidades de aprendizaje e intervención educativa.

Estas articulaciones garantizan un currículo interdisciplinario que coordina los espacios curriculares relacionados con los contextos reales. La sistematización de este proceso formativo experiencial se integra con los proyectos de vinculación con la comunidad y finaliza con el trabajo de titulación.

La exploración de la realidad nacional en Ecuador a la hora de diseñar la carrera de Educación Especial con enfoque inclusivo permitió constatar una formación docente asociada a titulaciones heterogéneas, currículos con escasos procesos de validación y actualización de los conocimientos de la ciencia, la profesión y la investigación, con modalidades de trabajo de titulación que no garantizaban calidad para logro del perfil de egreso de los futuros profesionales.

Por tal razón, se asumen los planteamientos de Larrea (2014, 2015) y Pérez Gómez y otros (2009) quienes declaran que la organización curricular debe garantizar procesos de formación, capacidades y competencias docentes que les permitan explicar y comprender los problemas de la realidad objeto de estudio o de intervención de la profesión; identificar problemas, tensiones y dilemas que definen los ejes del proceso de investigación; desarrollar habilidades de exploración, indagación, organización, explicación, estructuración e implicación con los problemas de la realidad investigada y objeto de transformación.

\section{La integración curricular en la carrera}

Los campos de formación epistemológicos, de investigación y de la praxis profesional están complementados de manera flexible para lograr el perfil de egreso deseado.

Estos campos de estudio se vinculan con los diferentes ejes de organización curricular:

- Núcleos problémicos (eje vertical): los sujetos, contextos y sistemas socioeducativos; el qué y cómo enseñar; los ambientes, procesos y resultados de aprendizaje; las necesidades específicas, adaptaciones y procesos de aprendizaje. Mediante estos núcleos se fortalecen los estudios de inclusividad.

- Ejes temáticos integradores por niveles (eje horizontal en la malla curricular): cada nivel de estudio tiene un eje temático que integra las asignaturas del ciclo. Estos son: aproximación a la política pública en educación; contextos familiarescomunitarios y aprendizaje de los sujetos educativos; modelos curriculares contextualizados; diseño, aplicación y evaluación de modelos pedagógicos y curriculares y la sistematización de la práctica de investigación-intervención educativa. 


\section{El modelo de prácticas pre-profesionales}

Las prácticas pre-profesionales complementan la formación del estudiante como un continuo de lo que acontece en el aula universitaria, atendiendo a diferentes aristas: sistemas educativos ante la diversidad, proyectos pedagógicos inclusivos; modelos curriculares contextualizados, flexibles y adaptados; diseño y gestión de ambientes de aprendizaje y procesos de identificación de necesidades educativas.

Las prácticas se organizan desde el primer ciclo de formación. Los tiempos fluctúan desde una vez por semana hasta ciclos de dos a ocho semanas de inmersión en las diferentes instituciones educativas.

Por consiguiente, en la UNAE, las prácticas pre-profesionales buscan desarrollar la gestión del conocimiento y los aprendizajes, en interacción con la innovación para que ofrezcan soluciones a las prioridades educativas locales y nacionales.

La carrera de Educación Especial con enfoque inclusivo está organizada curricularmente en tres unidades desplegadas en los nueve ciclos de trayectoria de los estudiantes: La unidad de formación básica, la unidad formación profesional y la unidad de especialización. La unidad de formación básica transcurre en los tres primeros ciclos y se centra en identificar y fortalecer las metodologías para conocer problemas, escenarios y contextos educativos con el fin de iniciar el proceso de inserción y diagnóstico. Luego, en la unidad profesional, desde cuarto hasta el sexto ciclos, se desarrollan los métodos de investigación, planificación, ejecución y evaluación de metodologías, modelos y programas educativos y pedagógicos para solucionar problemas y conflictos de los contextos inclusivos y especializados. Finalmente en la unidad de especialización, desde séptimo a noveno ciclos, se sistematizan experiencias de aprendizaje en contextos educativos, inclusivos y especializados, no escolares y escolares, a partir de un diagnóstico y un análisis sociocrítico y diferencial, para elaborar propuestas de adaptaciones curriculares y pedagógicas específicas para cada una de las especializaciones.

La práctica se organiza en torno a casos, problemas y proyectos; a partir de la integración de la teoría y la práctica. Desde el primer ciclo hasta culminar la carrera viene acompañada de reflexiones, conversaciones, lecturas, discusiones en clases y otras estrategias -usando diversas formas de agrupamiento- que permiten comprender su quehacer profesional y le brinda herramientas para desempeñarse mejor en sus funciones.

La formación práctica se orienta desde la perspectiva de aplicar lo aprendido en el aula universitaria para la solución de problemas profesionales. Se transita desde la familiarización hasta las inmersiones en las instituciones educativas. Se dirigen procesos de inclusión durante los seis primeros ciclos de formación en instituciones de la educación inicial y básica. A partir del séptimo ciclo se enfocan en los procesos de inclusión de niños y jóvenes portadores de necesidades educativas intelectuales, sensoriales y del lenguaje que asisten a instituciones especializadas.

La indagación, la investigación, la experimentación, la acción y la reflexión sobre la acción constituyen estrategias metodológicas de este modelo. La investigación-acción cooperativa, desarrollada mediante el modelo japonés de lesson study, constituye una de las estrategias privilegiadas tanto en las disciplinas teóricas como en el componente práctico del currículum de formación. 
La unidad de formación profesional tiene una mayor interacción en contextos reales y se enfoca en especificidades curriculares y de aprendizaje, planificación, inclusión y de necesidades especiales. Tiene como objetivo la intervención educativa para mejorar los modelos pedagógicos y curriculares a través de la enseñanza y ambientes de aprendizajes personalizados, interculturales e inclusivos.

Finalmente, en la unidad de Titulación se evalúan estos temas y se reflexiona sobre la intervención y la investigación educativa en los campos específicos de cada especialidad: necesidades educativas intelectuales, sensoriales y del lenguaje.

Las prácticas pre-profesionales configuran experiencias de formación para el trabajo que articulan las lógicas de acción de la formación académica con la vida profesional. Constituyen oportunidades de aprendizaje de inestimable valor que complementan el desarrollo curricular y aproxima a los estudiantes al universo material, simbólico e imaginario de la profesión.

Desde esta perspectiva, el aprendizaje en la carrera se entiende como el poder actuar con los saberes de manera flexible y en situaciones de índole profesional. Para esto, es preciso que el estudiante se aproxime a comprensiones más profundas, desarrolle en la práctica competencias para su quehacer, pueda actuar ante problemas profesionales reales, a partir de la intuición, los saberes previos y utilice herramientas teórico-conceptuales y prácticas; a partir de un proceso permanente de reflexión individual y grupal.

\section{La metaevaluación del desempeño profesional}

El proceso de formación a través de la práctica se caracteriza por su sistematicidad. Al finalizar cada jornada el profesor tutor se reúne con sus estudiantes para hacer una valoración de su desempeño y se exponen los logros y dificultes presentados. Esto crea las bases para los procesos reflexivos que se desarrollan en los proyectos integradores de saberes de cada ciclo y que tributan al proyecto de titulación de la carrera. Permite la consolidación de los procesos metacognitivos, pues los capacita para la reflexión acerca de su aprendizaje y el mejoramiento de su labor docente. (Arguelles y Nagles, 2007; Carretero, 2001; Flavell, 1976).

\section{Experiencias en la formación de docentes para la educación inclusiva, vivir las prácticas}

En correspondencia con las unidades de formación, el estudiante debe: explorar con enfoque diagnóstico la política pública; diagnosticar a los sujetos en sus contextos socioeducativos y a los problemas de enseñanza y aprendizaje -generales y específicos-; así como, diseñar y dirigir intervenciones educativas interculturales e inclusivas.

Desde la experiencia vivida en la interacción con los educandos y en la reflexión acerca de sus prácticas, se puede afirmar que se cuenta con un grupo de estudiantes altamente motivados por la profesión elegida y con un claustro conformado por 14 Doctores en Ciencias $(\mathrm{PhD})$ que presentan un alto nivel de exigencia para elevar los estándares de calidad en la UNAE.

Se mantiene un proceso de comunicación constante con los estudiantes para identificar necesidades de formación; a las cuales se les da respuesta a partir del diseño de programas 
de inducción para las prácticas pre-profesionales, con talleres y charlas que abarcan temáticas relacionadas con: elementos de didáctica general, el currículo ecuatoriano, adaptaciones curriculares, currículo diversificado, políticas educativas ecuatorianas, didácticas particulares e intercambio con expertos internacionales y nacionales en educación inclusiva.

Se incorporan los resultados de los proyectos de investigación de la carrera a las prácticas en el aula universitaria. Estos resultados incluyen la modelación del deber ser de la escuela inclusiva desde la realidad ecuatoriana; las buenas prácticas en la identificación de barreras para el aprendizaje y la participación en el aula; así como, el diseño de apoyos inclusivos y estrategias de diversificación curricular que han resultado innovadoras.

Los estudiantes, durante las prácticas, se organizan por dúos en las aulas y luego se agrupan en equipos de cuatro y seis para desarrollar las Lesson studies de forma cooperativa. Estas experiencias se socializan a la comunidad universitaria mediante jornadas científicas; a través de ponencias, poster y mesas redondas.

La organización de los proyectos integradores de saberes responde a las unidades de formación por las que transita el estudiante. La selección de los temas de los proyectos surge en un proceso inductivo-deductivo, donde acompañados por los tutores académicos y profesionales, identifican una problemática alrededor de los actores y escenarios educativos en los que deben profundizar como: las políticas de inclusión educativa; la relación de los contextos escuela, familia, comunidad; el diseño y desarrollo de proyectos pedagógicos inclusivos y las metodologías didácticas.

En la tutoría de los proyectos integradores de saberes, la triada pedagógica compuesta por los profesores de Investigación, Aproximación Diagnóstica y Cátedra Integradora se apoyan mutuamente orientando la reflexión sobre la práctica pre-profesional, con la participación del resto de los docentes del ciclo, a partir de casos, situaciones y problemas. Se proponen soluciones a las problemáticas de la escuela de acuerdo al contexto. Los directivos y tutores profesionales propician un diálogo de saberes entre la escuela y la universidad.

Esta triada pedagógica comparte tiempo y espacio para planificar, desarrollar y evaluar los procesos de formación docente. Además, orientan a los estudiantes a que reflexionen en torno a las situaciones educativas vividas en la práctica, basados en el principio pedagógico de experimentar la teoría y teorizar la práctica.

\section{Conclusiones}

La educación inclusiva se considera una condición indispensable para lograr un sistema educativo de calidad. Se asocia a la capacidad de ofrecer las mismas oportunidades de formación y desarrollo a todo el alumnado, a partir de los principios de equidad e igualdad.

En el proceso de diseño e implementación de la carrera de Educación Especial con enfoque Inclusivo se tuvo en cuenta la importancia que tiene la formación de docentes que eduquen en la diversidad y conduzcan prácticas educativas inclusivas. Se parte de las demandas de la sociedad ecuatoriana y de los más recientes estudios acerca del estado de la formación del profesorado para la educación con orientación inclusiva, como los realizados por: Mitler y Daunt (1995), Hegarty (1995), Forlin (2010, 2012), Donnelly y Watkins (2011); 
EADSNE (2011); Engelbrecht (2013) que reclaman una nueva identidad para sus profesionales, en torno a la ideología de la diversidad.

Los referentes teóricos consultados permitieron identificar dos posiciones en la formación de docentes para la Educación Especial. El enfoque formativo tradicional que forma profesionales que le asignan un valor preponderante al diagnóstico de las dificultades y a la derivación de los estudiantes hacia programas diferenciados o escuelas especiales y el enfoque inclusivo que busca formar docentes para que puedan responder a la diversidad de estudiantes en contextos lo más regulares posibles.

La formación de los profesionales para la educación inclusiva debe ser considerada como un espacio de reformulación, análisis y reconstrucción. Está vinculada a todo un proceso de cambio educativo que se realiza en la educación y que responde al enfoque de atención a la diversidad.

Las experiencias vividas, durante el proceso de implementación de la malla, han permitido recoger un grupo de evidencias que hablan a favor de lo pertinente del proceso formativo. Se ha logrado formar a un estudiante reflexivo e innovador en la búsqueda de soluciones a las problemáticas del aula de clase, con competencias para el trabajo colaborativo, en el diseño de prácticas basadas en la indagación, en la búsqueda conjunta de soluciones, el diálogo crítico y la confrontación reflexiva entre el profesorado de la escuela y en el diseño e implementación de propuestas que crean nuevos y variados escenarios para el trabajo con la diversidad en el aula.

\section{Referencias}

Abendaño Briceño, A. (2014). Procesos de formación de los docentes por parte de universidades e institutos pedagógicos en Ecuador. Recuperado de http://unesdoc.unesco.org/images/0014/001404/140489s.pdf

Ainscow, M. (2007). Taking an inclusive turn. Journal of Research in Special Educational Needs, 7(1), 3-7. https://doi.org/10.1111/j.1471-3802.2007.00075.x

Ainscow, M. (2015). Towards self-improving school systems. Londres: Routledge.

Ainscow, M. y Sandill, A. (2010). Developing inclusive education systems: The role of organizational cultures and leadership. International Journal of Inclusive Education, 14(4), 401-416. https://doi.org/10.1080/13603110802504903

Argüelles, D. y Nagles, N. (2007). Estrategias para promover procesos de aprendizaje autónomo. Bogotá: Alfaomega.

Blackhurst, A., Boot, D. y Cross, D. (1987). Non-categorical special education personnel preparation. En M. Wang, M. Reynolds y H. Walberg (Eds.), Handbook of special education: Research and practice (pp. 313-329.) Oxford: Pergamon Press.

Blanco, R. y Duk, C. (2011). Educación inclusiva en América Latina y el Caribe. Aula, 17, 37-55.

Carretero, M. (2001). Metacognición y educación. Buenos Aires: Aique.

Cuomo, N. (1994). ¿Dificultades de aprendizaje y/o enseñanza? Madrid: Paidós.

Donnelly, V. y Watkins, A. (2011). Teacher education for inclusion in Europe. Prospects, 41, 341353. https://doi.org/10.1007/s11125-011-9199-1

EADSNE. (2011). Formación del profesorado para la educación inclusiva en Europa. Retos y oportunidades. Odense: European Agency for Development in Special Needs Education. 
EADSNE. (2012). Formación del profesorado para la educación inclusiva. Perfil profesional del docente en la educación inclusiva. Odense: European Agency for Development in Special Needs Education.

Engelbrecht, P. (2013). Teacher education for inclusion, international perspectives. European Journal of Special Needs Education, 28(2), $115-118$. https://doi.org/10.1080/08856257.2013.778110

Feiman-Nemser, S. (1990). Teacher's preparation: Structural and conceptual alternatives. En W. R. Houston (Ed.), Hanbook of research on teacher education. Nueva York, NY: Mac Millan.

Flavell, J. H. (1976). Metacognitive aspects of problem solving. En L. B. Resnik (Ed.), The nature of intelligence (pp. 231-235). Hillsdale, NJ: Erlbaum.

Florian, L. (2008). Special or inclusive education: Future trends. British Journal of Special Education, 35(4), 202-208. https://doi.org/10.1111/j.1467-8578.2008.00402.x

Florian, L. (2010). The concept of inclusive pedagogy. En G. Hallett y F. Hallett (Eds.), Transforming the role of the SENCO (pp. 61-72). Buckingham: Open University Press.

Florian, L. (2011). European Union universities and teacher preparation. En B. Lindsay y W. Blanchett (Eds.), Universities and global diversity: Preparing educators for tomorrow (pp. 192206). Nueva York, NY: Routledge.

Florian, L. (2013). Preparing teachers to work with everybody: A curricular approach to the reform of teacher education. FORUM: For Promoting 31-19 Comprehensive Education, 55(1), 95-102.

Florian, L. (2014a). La educación especial en la era de la inclusión. ¿El fin de la educación especial o un nuevo comienzo? Revista Latinoamericana de Educación Inclusiva, 7(2), 27-36.

Florian, L. (2014b). The Sage handbook of special education. Londres: Sage Publications.

Forlin, C. (2010). Teacher education for inclusion. Changing paradigms and innovative approaches. Londres: Routledge.

Forlin, C. (2012). Future directions for inclusive teacher education: An international perspective. Abingdon: Routledge.

Giroux, H. A. (2001). Cultura, política y práctica educativa. Barcelona: Graó.

Hardman, M. y McDonnell, J. (2008). Disability classification and teacher education. En L. Florian y M. McLaughlin (Eds.), Disability classification in education, issues and perspectives (pp. 153169). Thousand Oaks, CA: Corwing Press.

Hegarty, S. (1995). Teacher training. En OECD., Integrating students with special needs into mainstream schools (pp. 59-68). París: OECD.

Larrea de Granado, E. (2014). El currículo de la educación superior desde la complejidad sistémica. Ciudad de México: Mundo Real.

Larrea de Granado, E. (2015). Unidad curricular de titulación. Quito: CES.

Liston, D. P. y Zeichner, K. M. (1993). Formación del profesorado y condiciones sociales de la escolarización. Madrid: Morata.

Marcelo, C. y López-Yáñez, J. (1997). Asesoramiento curricular y organizativo en educación. Barcelona: Ariel.

Marcelo, C. (1995). Formación del profesorado para el cambio educativo. Barcelona: PPU.

Martínez Bonafé, J. (1995). El profesorado ante el tercer milenio. Cuadernos de Pedagogía, 240, 2328. 
Mitler, P. (1993, mayo). Teacher education for special educational needs. Comunicación presentada en Seminar on policy options for special educational needs. Londres: University of London.

Mitler, P. y Daunt, P. (1995). Teacher education for special needs in Europe. Londres: Cassell.

Moya, A. (2012). El profesorado de apoyo en los centros ordinarios. Nuevas funciones, nuevas. Revista Siglo XXI, 30(1), 71-88.

OCDE. (2010). Educating teachers for diversity: Meeting the challenge. París: OCDE.

OEI. (2010). 2021 metas educativas. La educación que queremos para la generación de los bicentenarios. Madrid: OEI.

Parrilla, A., Blanco, A. y Herrera, J. I. (2015). Estudio exploratorio sobre pertinencia y perfil de egreso para la carrera de educación especial con enfoque inclusivo. Ecuador: UNAE.

Parrilla, A. (1996). La formación de los profesionales de la educación especial y el cambio educativo. En P. Jurado (Eds.), Las necesidades educativas: Presente y futuro (pp. 45-67). Barcelona: Lofe Artes Gráficas.

Parrilla, A. (1997). La formación de los profesores de apoyo: cuestiones a debatir. En P. Arnaiz y R. de Haro (Eds.), 10 años de integración en España: Análisis de la realidad y perspectivas de futuro (pp. 489-508). Murcia: Servicio de Publicaciones de la Universidad de Murcia.

Parrilla, A. (2015). Tendencias en la formación de docentes de educación especial: Perspectiva internacional. Madrid: Agencia Española de Cooperación Internacional para el Desarrollo.

Payá, A. (2010). Políticas de educación inclusiva en América Latina. Propuestas, realidades y retos de futuro. Revista Educación Inclusiva, 3(2), 125-142.

Pérez Gómez, A. (1992). La función y la formación del profesor/a en la enseñanza para la comprensión. Diferentes perspectivas. En J. Gimeno y A. Pérez Gómez (Eds.), Comprender y transformar la enseñanza (pp. 398-429). Madrid: Morata.

Pérez Gómez, A. (2010). Aprender a educar. Nuevos desafíos para la profesión docente. Revista Interuniversitaria de Formación de Profesorado, 24(2), 37-60.

Pérez Gómez, A., Soto E., Sola, M. y Serván Núñez, M. (2009). Contextos y recursos para el aprendizaje relevante en la universidad. Madrid: Akal.

Pullin, D. (2008). Implications for human and civil rights entitlements: Disability classification systems and the law of special education. En L. Florian y M. McLaughlin (Eds.), Disability classification in education (pp. 94-104). Thousand Oaks, CA: Corwin Press.

Riddell, S. (2014). A sociological perspective on special education. En L. Florian (Ed.), The Sage handbook of special education (pp. 97-109). Londres: Sage. https://doi.org/10.4135/9781446282236.n8

Shön, D. (1987). La formación de profesionales reflexivos. Hacia un nuevo diseño de la enseñanza y aprendizaje en las profesiones. Madrid: Paidós.

Shön, D. (1992). Formación de profesionales reflexivos. Barcelona: Paidos.

Spratt, J. y Florian, L. (2013). Aplicar los principios de la pedagogía inclusiva en la formación inicial del profesorado: de una asignatura en la universidad a la acción en el aula. Revista de Investigación en Educación, 11(3), 141-149.

UNESCO. (2009). Policy guidelines on inclusion in education. París: UNESCO.

UNESCO. (2012). Antecedentes y criterios para la elaboración de políticas docentes en América Latina y el Caribe. París: UNESCO.

UNESCO. (2015a). Revisión nacional de la educación para todos. Quito: UNESCO. 
UNESCO. (2015b). Foro mundial sobre la educación 2015. Incheon: UNESCO.

Vaillant, D. (2011). Preparing teachers for inclusive education in Latin America. Prospects, 41, 385398. https://doi.org/10.1007/s11125-011-9196-4

Vaillant, D. (2013). Formación inicial del profesorado en América Latina: Dilemas centrales y perspectivas. Revista Española de Educación Comparada, 22, 185-206. https://doi.org/10.5944/reec.22.2013.9329

Wang, M., Reynolds, M. y Walberg, H. (1987). Handbook of special education: Research and practice. Oxford: Pergamon Press.

World Health Organization. (2011). World report on disability. Ginebra: WHO.

\section{Breve CV de los autores}

\section{José Ignacio Herrera}

Doctor en Ciencias Pedagógicas (2000), Master en Ciencias de la Educación (1998) por la Universidad Central de Las Villas, Cuba, Licenciado en Psicología y Licenciado en Defectología, especialidad Logopedia. Catedrático de la Universidad de Sancti Spiritus de (1990 a 2014), miembro del Tribunal Permanente de Grado Científico de la República de Cuba. Profesor Principal I y Director de la carrera de Educación Inclusiva en la Universidad Nacional de Educación, Ecuador desde el 2015. Ha ejercido como profesor universitario por más de 30 años en el área de la Psicología y la Educación Especial como docente de pregrado y posgrado. Director de programas doctorales y de maestría. ORCID ID: 0000-0002-4750-3655. Email: joseighr2015@gmail.com

\section{Ángeles Parrilla}

Doctora en Pedagogía por la Universidad de Sevilla (1990), Licenciada en Ciencias de la Educación (Sección Pedagogía) y (Sección Psicología) en 1980 y 1982 respectivamente en la Universidad de Santiago de Compostela. Catedrática de la Universidad de Sevilla (2000 a 2011) y Universidad de Vigo (desde 2011). Directora del Doctorado Interuniversitario en equidad e Innovación en Educación, que se imparte en 5 universidades del noroeste español. Directora y fundadora del Grupo de investigación en Colaboración para la Inclusión Educativa y Social de la Universidad de Vigo. Ha participado y dirigido numerosos proyectos de investigación del campo de la Didáctica y Organización Escolar y los Procesos de Exclusión e Inclusión, Miembro del Comité de Dirección de la Escuela Internacional de Doctorado (EIDO) de la Universidad de Vigo. ORCID ID: 0000-00033393-6537. Email: parrilla.angeles@gmail.com

\section{Antonia Blanco}

Doctora en Filosofía y Ciencias de la Educación (1995) por la UNED, Licenciada Filosofía y Ciencias de la Educación (1988) por la UNED. Docente de la Universidad de Vigo en la Facultad de Ciencias de la Educación y del Deporte con más de 20 años de experiencia en la formación del profesorado, la innovación educativa, la evaluación institucional y el diseño de programas de programas de pregrado y posgrado. Ha trabajado como profesora de posgrado en la UNED-MEC, Nottingahm, Amsterdan y Alicante. Posee amplia experiencia en la formación de doctores en Ciencias. ORCID ID: 0000-0001-7797-9390. Email: antoniablancop2011@gmail.com 


\section{Geycell Guevara}

Doctora en Ciencias Pedagógicas, Máster en Ciencias de la Educación y Licenciada en Educación en la especialidad Español-Literatura por la Universidad de Sancti Spiritus, Cuba. Profesora no titular agregado 3 de la Universidad Nacional de Educación en Ecuador en el área de la didáctica de la lengua. Tiene 25 años de experiencia docente, de ellos 15 dedicados a la Educación Superior en programas de pregrado y posgrado. Ha participado y dirigido varios proyectos de investigación en temas de orientación educativa, tutoría, didáctica, educación inclusiva y redacción académica. ORCID ID:0000-00034872-8431. Email: geycellgf@gmail.com 